\title{
Understanding the evolution of radiation damage on the Gaia CCDs after 72 months at $\mathrm{L} 2$
}

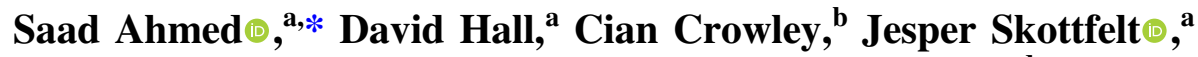 \\ Ben Dryer, ${ }^{\mathrm{a}}$ George Seabroke $\odot,{ }^{\mathrm{c}}$ Jose Hernandez $\odot,{ }^{\mathrm{d}}$ and \\ Andrew Holland ${ }^{\mathrm{a}}$
}

${ }^{a}$ The Open University, Center for Electronic Imaging, SPS, Milton Keynes, United Kingdom

${ }^{\mathrm{b}} \mathrm{HE}$ Space Operations BV for ESA/ESAC, Camino Bajo del Castillo s/n, Villanueva de la

Cañada, Spain

${ }^{c}$ University College London, Mullard Space Science Laboratory, Surrey, United Kingdom

${ }^{\mathrm{d} E u r o p e a n}$ Space Agency, European Space Astronomy Center, Camino Bajo del Castillo s/n, Villanueva de la Cañada, Spain

\begin{abstract}
The European Space Agency's Gaia spacecraft has been operating in L2 ever since its launch in December 2013 with a payload that includes 106 scientific charge-coupled devices (CCDs). Due to the predicted radiation environment at the pre-flight testing stage in addition to the high level of accuracy demanded by the science objectives, the non-ionizing energy loss (NIEL) damage on the detectors was identified as a major factor that could affect the science goals of the mission. Here, we present the analysis of an extended set of charge calibration data, taken up to almost six years after launch. It is found that the rate of radiation damage accumulation by the CCDs has not differed significantly from previous results. While the parallel and serial CTI measure an increase in time, the trap defect landscape is still dominated by the preflight defects rather than the radiation-induced traps. CCD devices that were predicted to have a lower NIEL dose measure comparatively larger rates of CTI increase. In addition to this, thicker devices have been measured to have lower serial CTI values compared to thinner devices. The initial parallel CTI values have also been found to be dependent on manufacture year. () 2022 Society of Photo-Optical Instrumentation Engineers (SPIE) [DOI: 10.1117/1.JATIS.8.1 $.016003]$
\end{abstract}

Keywords: Gaia; charge-coupled devices; European Space Agency; radiation damage; protons; charge transfer inefficiency.

Paper 21042 received Apr. 9, 2021; accepted for publication Jan. 14, 2022; published online Feb. $1,2022$.

\section{Introduction}

The Gaia spacecraft is an European Space Agency (ESA) medium-class mission from the Horizon 2000 Plus program, successfully launched in December 2013. Over its mission, it will observe and take measurements of more than one thousand million stars with the goal of making the largest catalog of astronomical objects in the Milky Way. Rotating with a period of $\sim 6 \mathrm{~h}$, Gaia scans the sky with its two optical telescopes, making repeated observations of stars, galaxies, quasars, and other objects that are autonomously detected onboard. In addition to astrometric parameters, Gaia data releases (DRs) contain (or will contain) photometric results, lowresolution spectra, colors, and astrophysical parameters of most stars in addition to radial velocities and medium-resolution spectra of a subset of objects. ${ }^{1,2}$ As of the time of this publication, two complete DRs and an Early third DR have already been released based on the large quantities of data collected by the spacecraft ${ }^{2,3}$ with the full third DR and future DRs to be produced in the future (for the latest updates on the DRs and the mission in general see Ref. 4). Gaia has a focal plane of 106 charge-coupled devices (CCDs), which continually make measurements of

*Address all correspondence to Saad Ahmed, saad.ahmed@open.ac.uk 


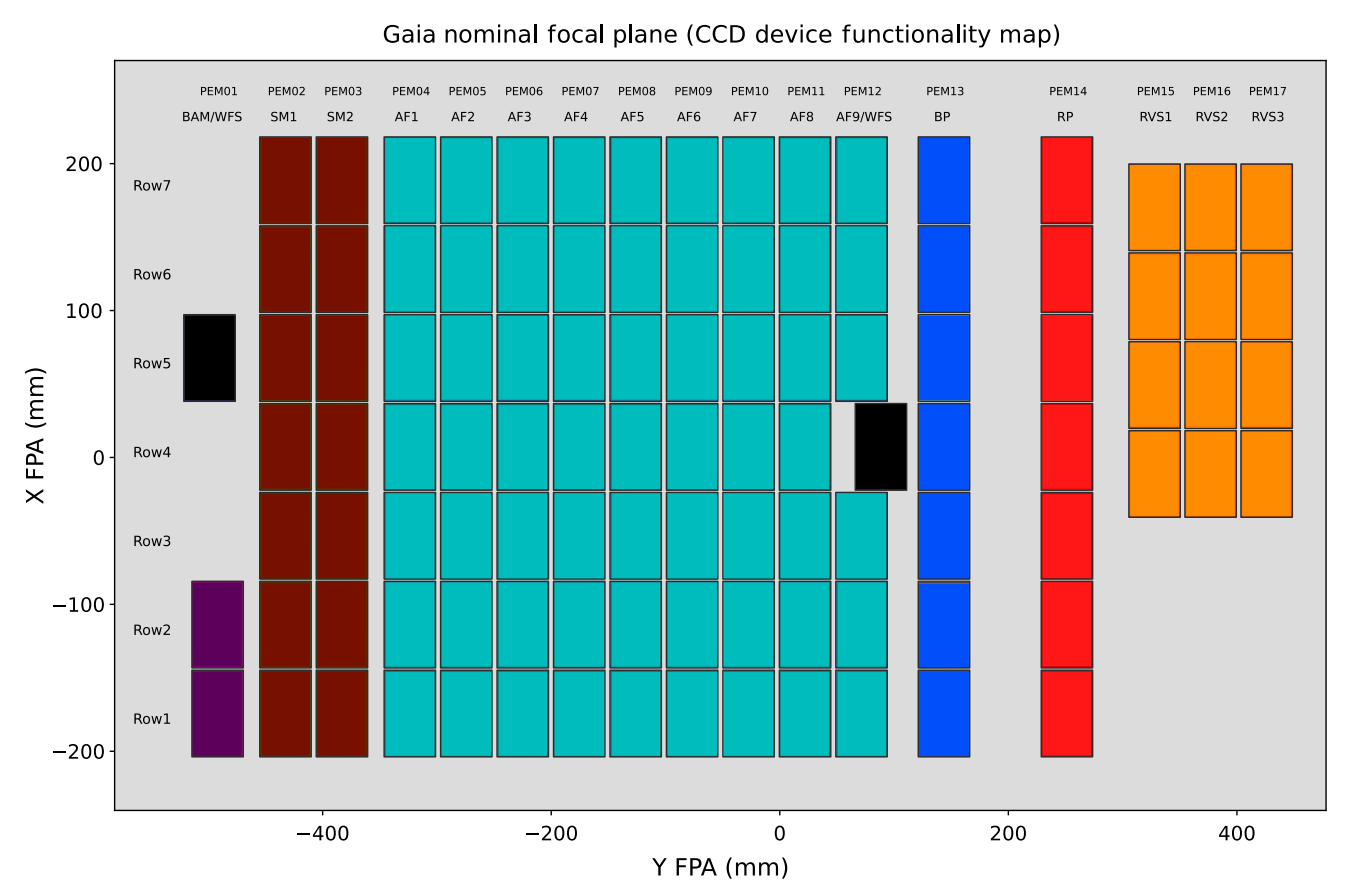

Fig. 1 The Gaia focal plane with 106 large-format CCDs. The CCDs are split into seven rows and 17 columns, each with a set of readout electronics known as proximity electronic modules. The devices all run in synchronisation with the aid of an on-board computer. The CCDs are also split into 17 different strips. The CCDs in this figure are color-coded according to the functional group they belong to. The color designations are as follows: black for the wavefront sensors (WFS), purple for the BAM, maroon for the SM, turquoise for the AF, blue for the BP, red for the RP, and orange for the RVS. Figure reproduced from previous work. ${ }^{5}$

objects in its fields of view. Figure 1 shows a schematic of the focal plane where the CCDs are color-coded by their instrument. Due to the continually scanning nature of the mission, the CCDs operate in time delay and integration (TDI) mode, transferring charge at the same rate as the objects move across the detectors. ${ }^{2}$

Gaia orbits Lagrange Point 2 (L2), the same destination of several other future space missions, such as ESA's PLAnetary Transits and Oscillations of stars, Atmospheric Remote-sensing Infrared Exoplanet Large-survey and Euclid missions, NASA's Roman Space Telescope as well as NASA and ESA's James Webb Space Telescope. Due to the predicted radiation environment in this region at the time of Gaia's pre-launch phase, as well as the high-precision measurement requirements of the mission, the increase of charge transfer inefficiency (CTI) in the CCDs from non-ionizing radiation damage was identified in the pre-launch studies as a major factor that would impact the useful scientific output of the mission. ${ }^{5}$ As a result, many tests and radiation campaigns were conducted during the pre-flight stage to quantify the impact of radiation damage, and several CTI mitigation strategies were devised. ${ }^{6,7}$ However, a comparatively more recent analysis of in-flight data $\sim 30$ months after launch has revealed that Gaia is predicted to accumulate roughly an order of magnitude less damage than was initially expected during its nominal lifetime. ${ }^{8,9}$ In fact, the overall spacecraft performance has been so good that Gaia's mission time has been extended past its original five-year plan. Although this is good news for the science goals of the mission, it is important to understand, as much as possible, the reasons behind the differences between the predicted and measured results to better understand the physics and mechanisms for more accurate testing and predictions for other missions.

This paper analyzes an additional set of in-flight data, now taken up to six years after its launch date, to examine the on-board radiation damage (using specially acquired calibration datasets) and check whether there have been any major deviations from the behavior measured in 2016. A previous analysis only looked at the CTI between two different points in the mission. 
This paper is an extension of the previous work and analyses the data across multiple points in the mission and quantifies the evolution and increase of the CTI in time. ${ }^{5}$

\section{Radiation Damage in CCDs}

CCDs have a rich history of use in many space applications and missions in the past. CCDs consist of a metal-oxide-semiconductor structure where charge in the form of photo-electrons is generated in the semiconductor from incident photons. In space, CCDs aboard spacecraft are exposed to radiation, which consists of energetic particles, such as protons, electrons, and neutrons from galactic cosmic rays (GCRs) and solar events. These particles can cause displacement damage in the CCDs.

When displacement damage occurs, atoms are knocked out of the silicon lattice by the high energy particles. The exact spectrum and energy of particles varies with respect to orbit, location in space, and the behavior of the Sun. The vacancy, or absence of the silicon atom, diffuses through the silicon and can form a stable defect with either another vacancy or an impurity atoms, such as oxygen or phosphorus. These defects, or traps, have their own characteristic energy levels and the traps capture and emit photo-electrons from passing charge packets on probabilistic timescales. Depending on the charge transfer period, trails of charge can be formed behind the signal packets, which reduces the quality of the data and distorts the signal. ${ }^{10}$

\section{Gaia CCD Details}

Gaia's CCDs, which have been custom-designed and manufactured by Teledyne e2v, have a number of special features to reduce the impact of radiation damage and charge trapping. The CCDs were designed with a supplementary buried channel (SBC), which is a notch in the buried channel region that can confine small charge packets to a smaller volume so that they encounter fewer traps. In addition to this, the CCDs have a charge injection (CI) gate structure where rows of charge can be periodically injected and transferred through the CCDs to keep traps filled so signals can pass by undistorted. ${ }^{11}$ While all of Gaia's CCDs were manufactured with these features and with identical chip architectures, they were also manufactured in three different variants, each optimized to improve their quantum efficiency in different wavelength regimes to fulfill different objectives.

\subsection{Device Variants and Year of Manufacture}

About 78 of the focal plane CCDs were of the astrometric field (AF) variant device, which were built on $25-\mu \mathrm{m}$ standard silicon, thinned to $16 \mu \mathrm{m}$ with a resistivity of $100 \Omega \mathrm{cm}$, and have an anti-reflection (AR) coating centered at $650 \mathrm{~nm}$. These devices were implemented on the AF and Sky Mapper (SM) instruments as well as the wave-front sensors (WFS). The seven CCDs of the Blue Photometer (BP) instrument used the BP variant CCDs, which were made with $16 \mu \mathrm{m}$, $100 \Omega \mathrm{cm}$ resistivity standard silicon but had an AR-coating that was centered on $360 \mathrm{~nm}$, making them more sensitive to bluer wavelengths. The third CCD variant was the red photometer (RP) variant that was used on instruments that required greater sensitivity to redder wavelengths. They were manufactured with a thickness of $40 \mu \mathrm{m}$ using deep-depleted silicon that had a resistivity of $1500 \Omega \mathrm{cm}$ and had an AR-coating centered at $750 \mathrm{~nm}$. These variants were used in the seven CCDs of the RP Instrument, the 12 CCDs of the radial velocity spectrometer (RVS), and the two basic-angle monitor (BAM) CCDs. ${ }^{5,8,12}$

Many different devices were manufactured in the time before launch, to be used in performing radiation testing and sector analysis or to be implemented as in-flight devices. Due to the comparatively larger number of devices required on the focal plane as compared to other space missions, the in-flight devices were all manufactured at different times and came from different batches of silicon. In a previous study, it was found that the CCDs that were manufactured after 2004 were more likely to contain a reduced SBC full well capacity as compared to earlier devices. ${ }^{13}$ To check for any correlations between device performance in the results of this paper 


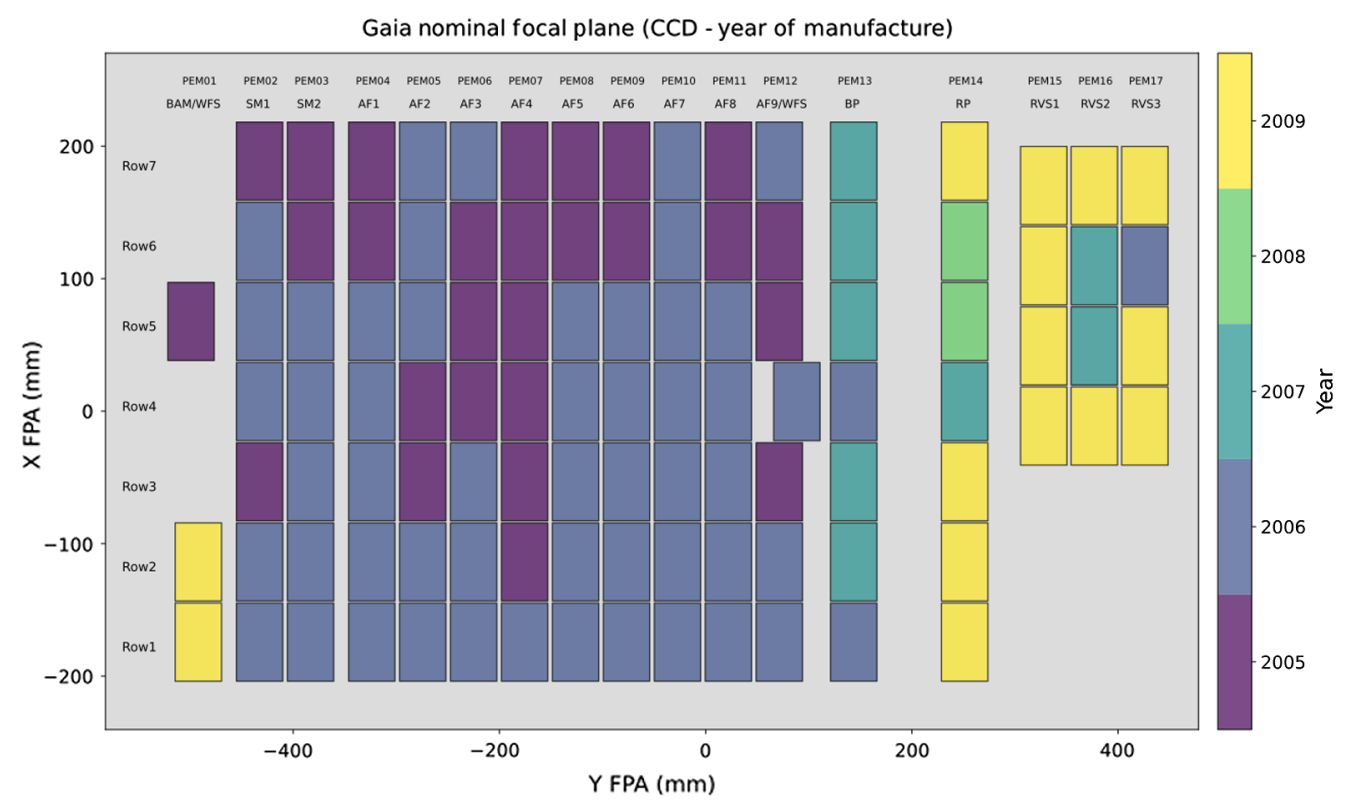

Fig. 2 The year of manufacture of all the in-flight devices on the Gaia focal plane; reproduced from previous work. ${ }^{5}$

and the time of production, Fig. 2 was produced, which illustrates the corresponding year of manufacture of the in-flight devices. ${ }^{5}$

\subsection{Pre-Flight Tests}

As has been mentioned previously, a number of pre-flight tests were conducted on the Gaia CCDs to work out how to optimize the CCD performance as well as to investigate the effects of radiation damage. Surrey Satellite Technology (formerly Sira) and Airbus Defence and Space (formerly EADS Astrium) were the main industrial partners of the mission who performed these tests, the latter of whom performed five radiation campaigns to test device performance. While hardware optimization structures were implemented into the design, as outlined previously, operating techniques were also investigated, which would optimize the performance as much as possible. Some of the main conclusions derived from these campaigns were selecting a $163 \mathrm{~K}$ operating temperature to optimize CTI between the parallel and serial directions, the use of periodic CIs to reset the illumination history in the CCDs, and the rejection of artificial light to produce flat-fields for calibrations. ${ }^{6,12}$

A number of computer models and simulations were also used in conjunction with these experimental tests. To measure the CCD irradiation levels to be used in the radiation campaigns, Airbus Defence and Space simulated a prediction for the $10-\mathrm{MeV}$ equivalent NIEL dose of protons across the focal plane; Fig. 3 reproduces this predicted NIEL dose. ${ }^{14}$ This prediction was made in 2006, using the predicted level of solar activity at the time. This NIEL dose prediction only considered particles from solar events, neglecting the effects of GCRs, as well as only considered the primary emission of particles. In Fig. 3, it can be seen that the AF devices receive the largest fluence of 10$\mathrm{MeV}$ equivalent protons in this prediction, with the devices in the center receiving the largest dose due to these devices being exposed the most to radiation. The BP, RP, and RVS instruments have optics and photometers setup in front of the devices which causes a reduced impact from the 10$\mathrm{MeV}$ equivalent dose, as is noted in Fig. 3. This predicted pattern formed the basis for the irradiations used in all the pre-flight tests; because the AF CCDs were predicted to receive a dose of $3.1 \times 10^{9} \mathrm{p}^{+} / \mathrm{cm}^{2} 10-\mathrm{MeV}$ equivalent protons, irradiations were performed at a slightly higher level of $4 \times 10^{9} \mathrm{p}^{+} / \mathrm{cm}^{2} 10-\mathrm{MeV}$ equivalence to overestimate the damage. ${ }^{14,15}$

A semi-empirical model was also developed during the pre-flight testing called the charge distortion model (CDM) $;{ }^{16}$ it was developed for forward modelling to reproduce the image distortion from the effects of radiation damage. CDM uses an electron confinement volume model 


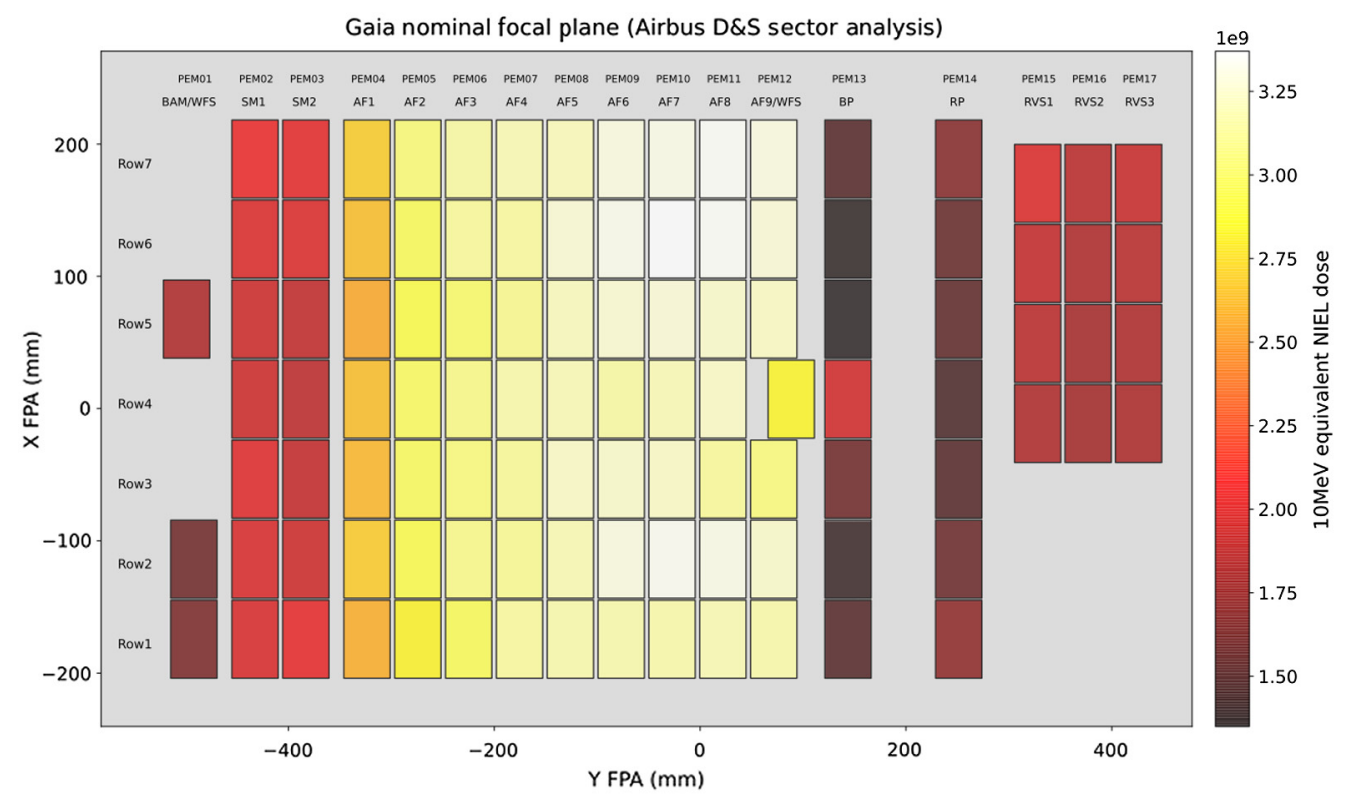

Fig. $3 \mathrm{~A}$ graphical representation of the $10 \mathrm{MeV}$ equivalent proton NIEL dose, measured in protons $/ \mathrm{cm}^{2}$, predicted by Airbus Defence and Space a number of years before launch in 2006. The EOM NIEL prediction for the CCDs ended up being lower than this at the time of launch but irradiation levels for the on-ground tests were based on this set of predictions.

where the signal level and the volume confined by the charge cloud are related by a power-law, defined by a $\beta$ parameter. ${ }^{16}$ Previous work has been successfully conducted to model observations with CTI to obtain the true image parameters from the damaged observations. ${ }^{6,7}$

\section{Charge Data Monitoring}

Calibration activities are run periodically on-board Gaia, initially with a cadence of every 3 to 4 months, to monitor the performance of the devices. Unique strategies are adopted to make measurements of different detector characteristics. Previous analyses of these measurements have revealed that the detectors are functioning as expected with no major issues. ${ }^{8,17}$

The CTI is measured regularly by examining the charge loss from CI lines. Previous analysis of both the in-flight parallel and serial [along-scan (AL) and across-scan (AC) respectively, using the Gaia terminology] CTI data in 2016 revealed that the radiation damage experienced by the inflight CCDs has been well below the predicted end-of-mission (EOM) 10-MeV equivalent fluence values and the degradation in performance has been less than expected. ${ }^{8,9}$ While this is beneficial for the science objectives of the mission, it is important to understand, as well as possible, why this discrepancy has occurred between the pre-flight tests and predictions and the in-flight data. This is to verify the reliability in the pre-flight testing as well as to obtain a better overall understanding of what is going on which can positively feedback into other missions and studies. The previous analysis of charge calibration data concluded that the lower than expected CTI levels are most likely the result of a lower solar activity level during solar cycle 24 as well as due to mitigation of charge loss from background straylight. ${ }^{9}$ There is potential work to be done to accurately quantify all the factors causing the differences between the inflight and on-ground results and the results of this paper provide a starting point from which to go forward.

This paper analyzes more in-flight data, using data taken all the way up to the end of 2019 , beyond the original EOM date, to see how the CTI level and radiation damage compares to the previous results. In previous studies, AL and AC CTI were measured from alternate CI schemes. Regular CIs are conducted periodically at fixed levels in the AF, BP, and RP devices; these are carried out every $\sim 2 \mathrm{~s}$ (corresponding to a 2000 TDI period) for the AF devices and every $5 \mathrm{~s}$ 


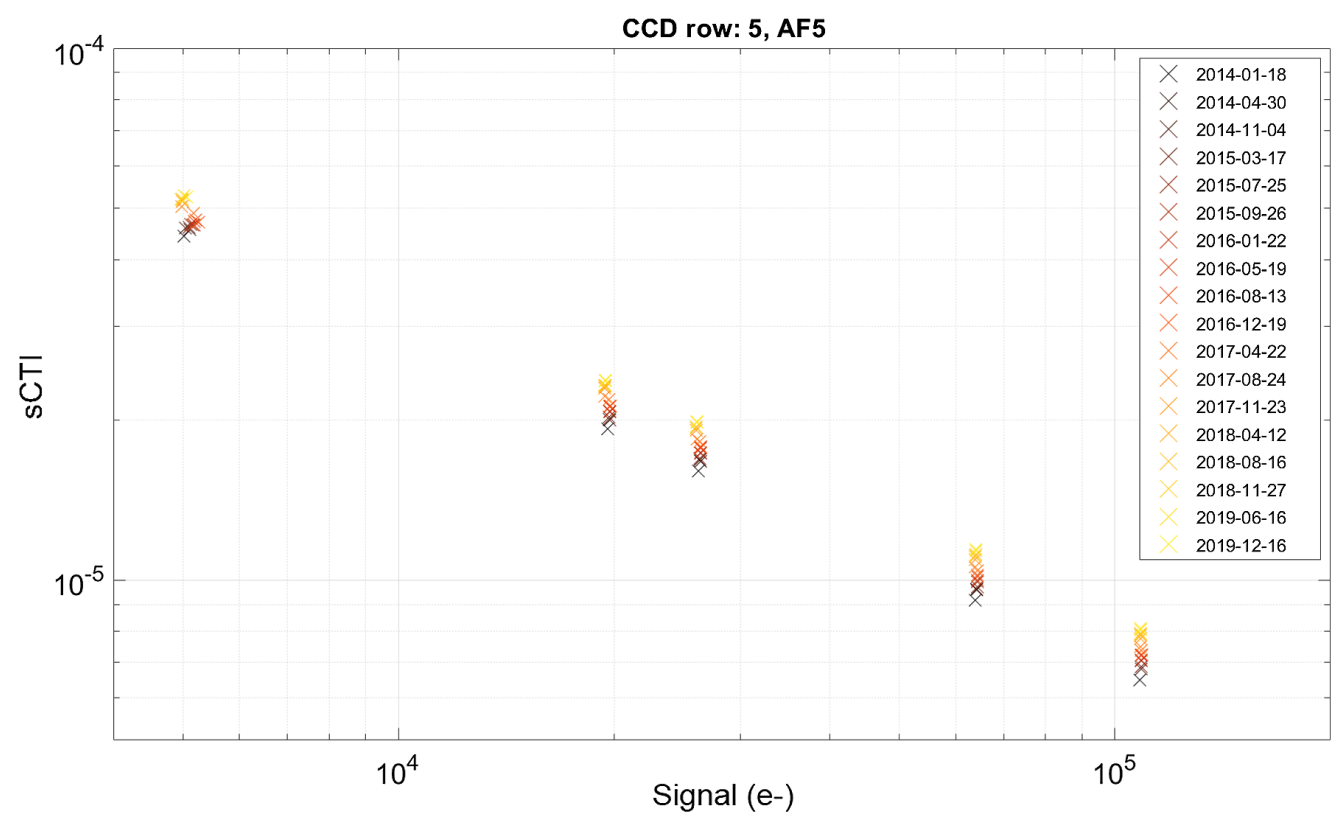

Fig. 4 The five $\mathrm{Cl}$ values with corresponding $\mathrm{CTI}$ values for each calibration run for a device near the center of the focal plane. A slow but finite degradation in $\mathrm{CTI}$ is visible with respect to time. For the sake of completeness, data from January 2014 is produced here; the data from this time are not used in the rest of this study as the focal plane was known to be at a higher temperature.

(5000 TDI) for the BP and RP devices. While the RVS devices were built with a CI structure, one that is used in some of the calibration activities, regular CIs are not implemented on these devices as the signal would interfere too much with the finer measurements and long spectral windows and the leading edge of the long spectrum would act to fill the traps. ${ }^{8}$

Compared to the CI period of 2000 TDIs in the AF CCDs and 5000 TDIs in the photometers, the CIs investigated in this study had a smaller period of 455 TDIs. This means that more traps would have been filled between the charge-injection generated virtual objects (VOs) and so the measured parallel CTI values will be lower as compared to the values measured with the regular CIs. The trends of the CTI behavior with time should still be clearly visible. In addition, the use of these datasets to measure parallel CTI means that parallel CTI can also be investigated in the RVS devices. As previous studies only looked at parallel CTI measurements from CIs, new insights are obtained about the behavior of the RVS devices. The CTI measurements can also be more easily compared between different devices because the CIs of the calibration activity all have similar charge levels and periods across all the devices as opposed to the regular CIs, which are not only non-uniform between CCDs, but, as mentioned previously, had different periods depending on the instrument. ${ }^{8,15}$

The technique that is used to analyze the CTI in the serial direction involves generating charge in the image pixels and monitoring any trailing in the post-scan pixels after transferring through the serial register. Five different levels of CI are generated in this activity with VOs placed over the end of the image area. This calibration activity was initially proposed to be run monthly or bi-monthly but due to the lower than expected amount of damage, it is now run every three to four months; it is likely to be less frequent in the future. ${ }^{8,15}$ Figure 4 details a set of serial CTI results with respective signal levels for a single CCD at all the different times the calibration activity was run. It can be seen that there is a small but finite increase of CTI with time. As the charge is generated at one end of the CCD and is transferred across the device to the serial register at the other end, it is possible to investigate the parallel CTI using this set of data as well. In this study, both the serial and parallel CTI were measured using just the calibration data; data were only available for science CCDs, except for the SM CCDs.

An important complexity should be noted about the CTI results. The parallel transfer speed is at the TDI scanning speed of $982.8 \mu$ s so the parallel CTI should be reflective of any trap species 
emitting around those timescales. In comparison, transfer in the serial direction takes place at two different speeds; there is a $10-\mathrm{MHz}$ flush to transfer data to the readout node and a slower $\mathrm{kHz}$ speed for readout. ${ }^{8}$ This means the charge tail will be formed from trap emission at two different timescales. For these results, only emission at $\mathrm{MHz}$ timescales is considered as it dominates the serial CTI but it is noted that it would be useful to conduct a study in the future to look at the differences between different readout speeds.

To obtain the next set of measurements and compare the CTI of all the devices against each other, a number of processing steps were conducted. A power-law relationship was fit between the parallel and serial CTI values and their corresponding CI levels. ${ }^{5}$ The reason a power law was chosen was due to a similar model used in Gaia's $\mathrm{CDM}^{16}$ and the verification of such a relationship from other experimental studies. ${ }^{18}$ Once this relationship was extrapolated, a CTI value was calculated for the same signal level $(10,000$ electrons was used for all the results) for all the devices. By using the same virtual signal, the CTI between all the devices can be more easily compared against each other. ${ }^{5}$

\section{Serial CTI (AC Direction)}

\subsection{4 and 2019 Data}

Using the data analysis steps outlined in the previous section, the diagrams in Fig. 5 were generated, which highlights the serial CTI of all the CCDs at two different times, initially computed and analyzed in a previous work. ${ }^{5}$ Figure 5(a) shows the CTI in April 2014, just a few months after launch, while Fig. 5(b) shows the CTI in December 2019, more than five years later. The mean CTI for each instrument was also calculated at the two times and these values are given in Table 1. The calibration data from January 2014 was not used as the cool-down of the payload was still not complete. Figure 5(a) is similar to the plot generated in the previous set of results in 2016, verifying the reliability of these results. It is assumed that the oxygen defect, called the Acenter, is likely to be responsible for the bulk of the serial CTI as it has a comparable release time with the 10-MHz flush that dominates the serial transfer. A previous pre-flight test revealed a trap species that emitted faster than the A-center so there is a chance for the AC CTI to be caused from this trap species as well. ${ }^{8,19}$

The 2014 results in Fig. 5(a) and Table 1 should be reflective of the initial CTI state of the CCDs as the accumulated radiation damage within the first few months is known to be minimal. The results show that the thicker RP devices, found in both the RVS and the RP, all measure a lower serial CTI as compared to the other devices. Even though thicker devices are known to have different properties to thinner devices, such as being more sensitive to cosmic rays and having larger amounts of dark current, it is currently unknown why these devices would be manufactured measuring a lower serial CTI compared to thinner devices with the same architecture. ${ }^{20}$ If the serial CTI is indeed primarily caused by A-centers, then this could be due to a smaller amount of oxygen and A-center defects being present in the buried channel region. This could have been the result of impurity oxygen having a larger amount of silicon to diffuse through during manufacture. It would be useful to perform trap-pumping on unirradiated thick and thin devices to understand the trap landscape and narrow down the reasons behind this behavior. Trap-pumping is an analysis technique that makes it possible to observe individual trap species and their properties with a high level of accuracy and quantify their densities. It involves the clocking of a flat-field signal between phases which leads to the formation of dipole maps that can be studied in more detail. ${ }^{21-23}$

It can be seen that the pattern of serial CTI distribution across the focal plane is the same in 2014 and 2019. This indicates that the serial CTI is still dominated by the initial CTI state and the amount of radiation damage has not been significant enough to alter this distribution pattern. The 2019 CTI values in Table 1 are within the same order of magnitude as the 2014 CTI values; this demonstrates a very low rate of increase. The AF and BP CCDs measure a smaller percentage increase in their serial CTI ( 6\% to 9\%) as compared to the red variant devices $(\sim 27 \%)$. ${ }^{5}$ With Gaia having been in orbit for just over its original mission lifetime, no major deviations are seen from the previous set of CTI results, as measured in 2016. 
Ahmed et al.: Understanding the evolution of radiation damage...
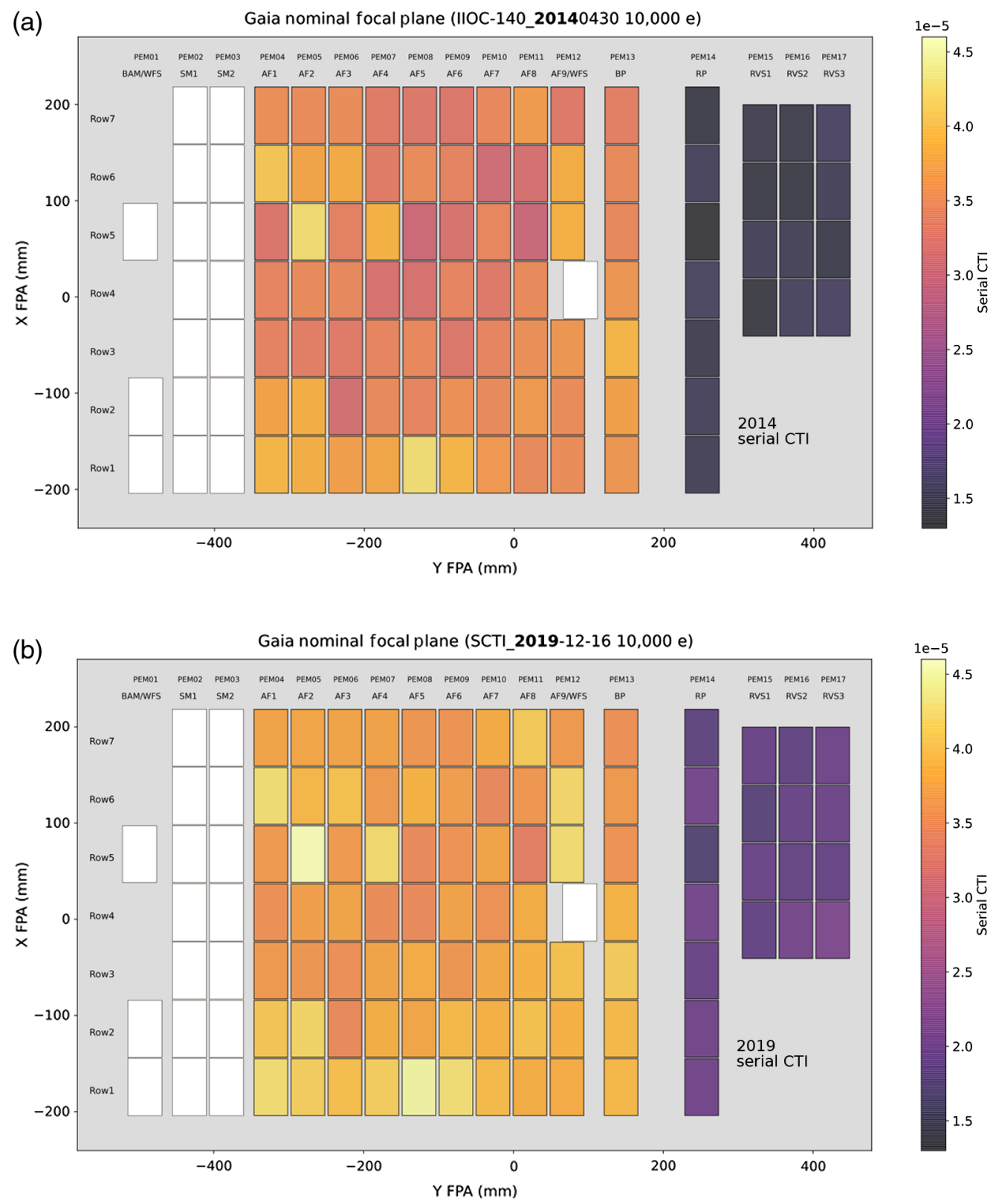

Fig. 5 The distribution of the derived serial CTI values across the focal plane for a signal of 10,000 electrons as measured in (a) April 2014 and (b) December 2019; reproduced from previous work. ${ }^{5}$

Table 1 Mean serial CTI (at 10,000 electrons) for each instrument as measured in April 2014 and December 2019.

\begin{tabular}{lcc}
\hline \hline CCD instruments & 2014 mean CTI $\left(\times 10^{-5}\right)$ & 2019 mean CTI $\left(\times 10^{-5}\right)$ \\
\hline $\mathrm{AF}$ & $3.5 \pm 0.3$ & $3.8 \pm 0.3$ \\
$\mathrm{BP}$ & $3.6 \pm 0.2$ & $3.8 \pm 0.2$ \\
$\mathrm{RP}$ & $1.5 \pm 0.1$ & $1.9 \pm 0.1$ \\
$\mathrm{RVS}$ & $1.5 \pm 0.1$ & $1.9 \pm 0.1$ \\
\hline \hline
\end{tabular}




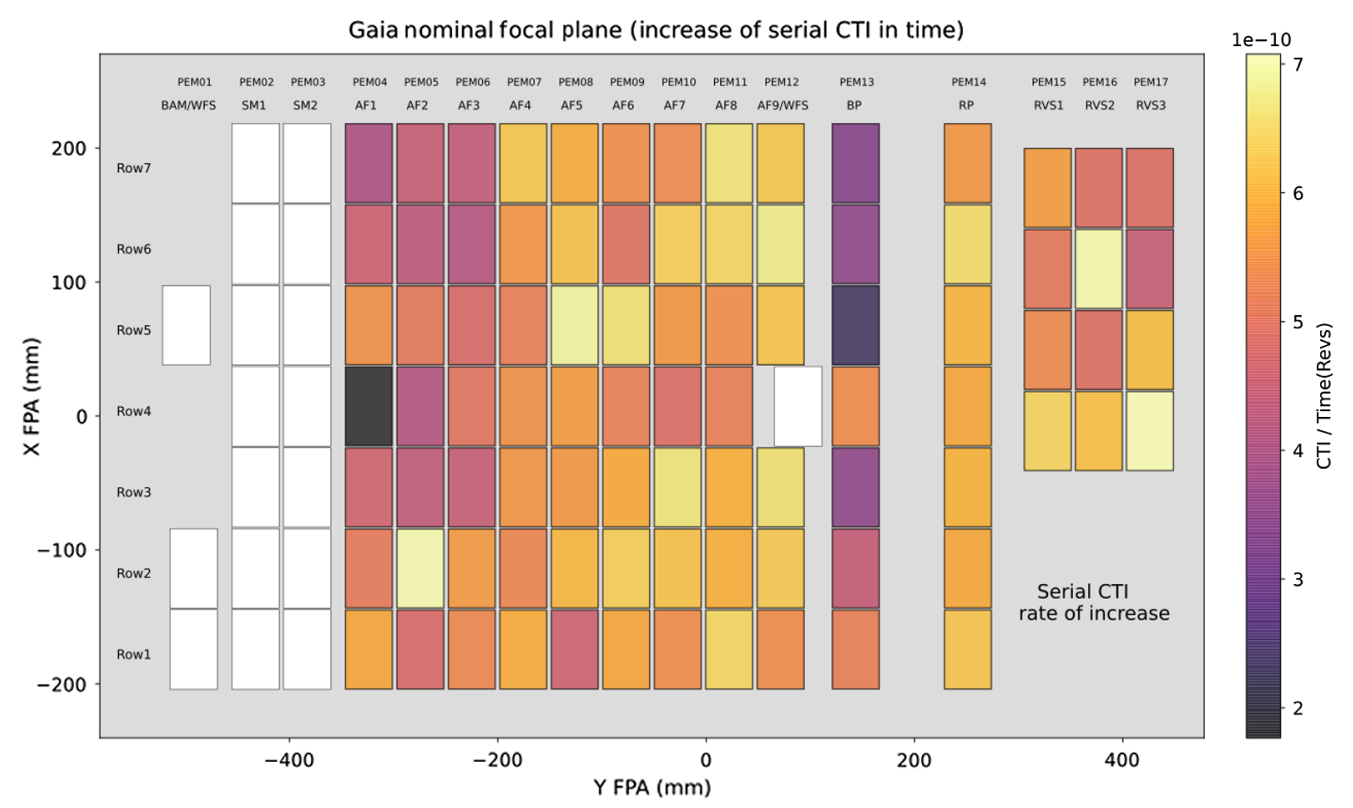

Fig. 6 The extrapolated rate of increase of serial CTI (at 10,000 electrons) for each CCD during the six-year period of the charge calibration.

\subsection{Rate of Increase}

Using the serial CTI values for each CCD at the time of each calibration activity, a linear relationship was fitted between serial CTI and time. The slopes derived from these relationships were taken as the rate of increase of CTI with time and were computed for each CCD; these values are shown for each CCD in Fig. 6. If outliers are ignored, the column-wise CTI distribution would seem loosely similar to the NIEL-dose prediction from Fig. 3, however, the pattern is not very clearly defined so a correlation cannot be established.

Combining the data from the CCDs for each instrument, the values in Table 2 are produced which gives the mean rate of increase for each instrument, in units of both Gaia revolutions and in years. Figure 7 shows the mean CTI for each instrument and how it increases with time; the values were calculated for each calibration activity run. Three major sets of solar flares are also marked out; the events in 2014 and 2015 had obvious effects in the previous set of data analysis of in-flight parallel CTI. ${ }^{9}$ Interestingly, these events do not seem to have a significant impact on the serial CTI. In contrast, the solar flare events in 2017, which were more larger and powerful, make a slightly more noticeable impact on this set of charge calibration data. This could be due to the relatively high level of CTI from the beginning of the mission which means the relative contribution from a solar event is much less evident on serial CTI. There is a loosely, linear increase of CTI in time, justifying the use of a linear extrapolation for the results of Fig. 6 and Table 2. The fluctuations and uncertainties seen in the data are likely due to photon noise from straylight and noise from bias and background subtraction during data processing.

Table 2 Mean increase in serial CTI per time for each instrument on the focal plane for a signal of 10,000 electrons.

\begin{tabular}{lcc}
\hline \hline CCD instruments & CTI increase/revs $\left(\times 10^{-10}\right)$ & CTI increase/year $\left(\times 10^{-7}\right)$ \\
\hline AF & $5.5 \pm 0.9$ & $8.0 \pm 1.0$ \\
BP & $4.0 \pm 1.0$ & $6.0 \pm 1.0$ \\
RP & $6.0 \pm 0.3$ & $8.8 \pm 0.4$ \\
RVS & $5.7 \pm 0.8$ & $8.0 \pm 1.0$ \\
\hline \hline
\end{tabular}




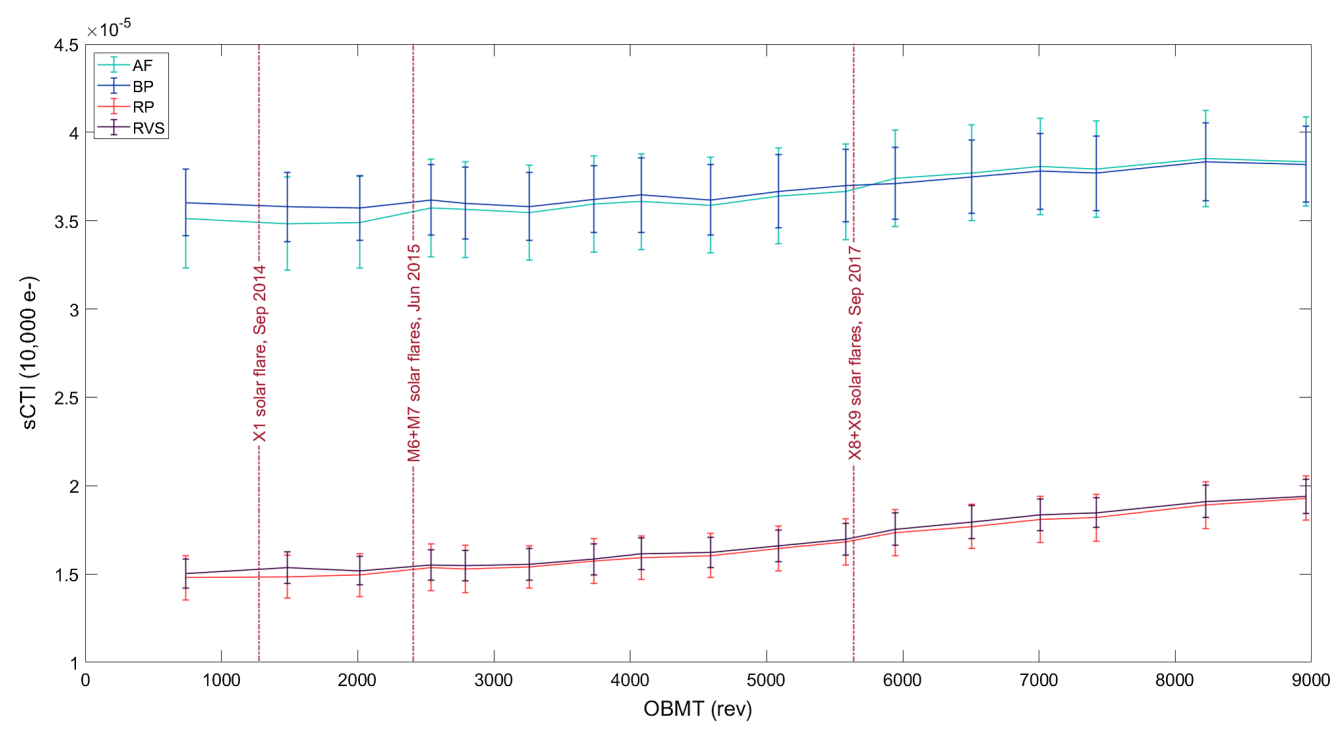

Fig. 7 Evolution of the mean serial CTI for each instrument, calculated from the CTI measured from all the devices at each different time. There is a steady, linear increase in time with fluctuations likely due to noise. Three main sets of solar flare events are all marked out. The $x$ axis spans a little under six years.

From the results of Figs. 6, 7, and Table 2, it can be seen that the RP devices in the RP and RVS measure comparatively higher rates of increases as compared to the other devices. It is known from Fig. 3 that the Blue and RPs receive an equivalent amount of fluence, likely due to similar levels of shielding. More tests will need to be conducted to more precisely determine the physical reasons behind the calculated higher increase. Current hypotheses include the initial CTI state or the geometry of the devices affecting the rate of radiation damage as well as interactions between the shielding and the radiation altering the energy spectrum of incoming particles.

\subsection{NIEL-Scaled Rate of Increase}

It is useful to track the evolution of CTI of each individual device to work out the correct data processing procedure for each CCD. However, by scaling the rate of increase results by the NIEL values from Fig. 3, it becomes possible to remove the variation in dose and shielding effects and examine the CTI increase between each CCD variant against each other more reliably. For example, in Fig. 6 and Table 2, it can be seen that there is a large variation in the CTI increase of the various AF devices due to the NIEL dose. Figure 8 reproduces Fig. 6 but with the rate of increase values of each CCD scaled by their corresponding NIEL dose value from Fig. 3 . Table 3 also shows the new rate of CTI increase values for each different instrument after the CCD values are scaled.

In Fig. 8, the AF devices now all highlight rates of CTI increase that are more comparable to each other as the shielding pattern from Fig. 3 is removed. This would indicate that all the AF CCDs experience radiation induced CTI increase on equivalent levels to each other. An interesting observation from Fig. 8 and Table 3 is that even after accounting for the NIEL dose, the RP devices still measure larger rates of CTI increase which are not measured by any of the other device variants. This gives more credence to the idea that it is a unique property of the thick RP devices that is causing this larger increase in CTI. It remains to be seen whether this feature is due to the lower initial CTI, an additional unknown effect of shielding not accounted for in the NIEL dose calculation, the manufacture of the devices, or due to device geometry.

It is noted from Fig. 8 that some of the BP devices seem to have rates of CTI increase that are more comparable to the RP devices than with the AF and BP devices. This would suggest that despite the reduced impact from the 10-MeV equivalent NIEL-dose, the shielding from the optics and photometers could somehow be affecting the radiation damage in a counterintuitively more 


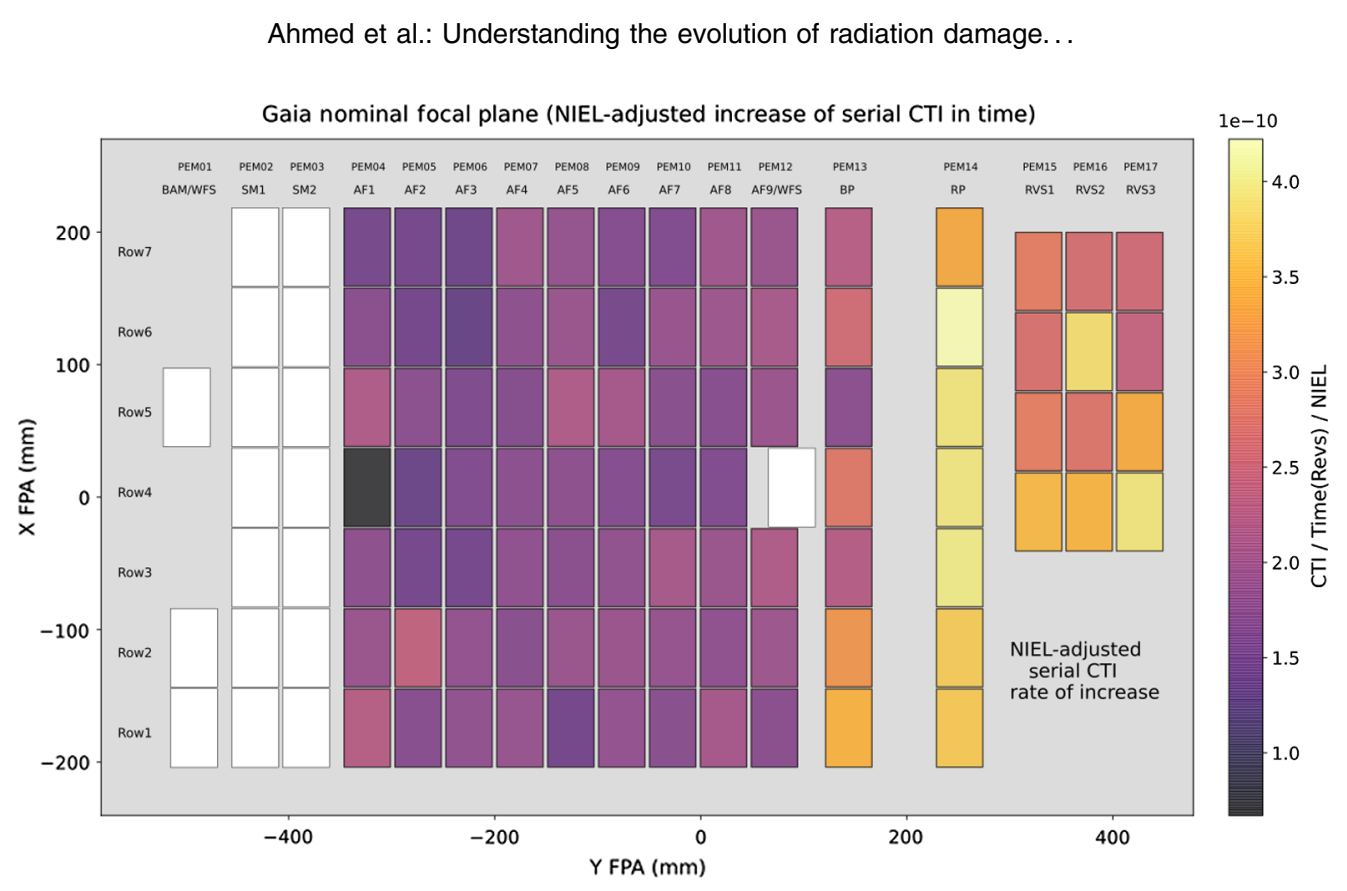

Fig. 8 The extrapolated rate of increase of serial CTI (at 10,000 electrons) for each CCD during the six-year period of the charge calibration data with each CCD scaled by its corresponding NIEL dose value from Fig. 3.

Table 3 NIEL-scaled mean increase of serial CTI per time for each instrument on the focal plane for a signal of 10,000 electrons.

\begin{tabular}{lcc}
\hline \hline CCD instruments & CTI increase $($ NIEL scaled $) /$ revs $\left(\times 10^{-10}\right)$ & CTI increase $($ NIEL scaled $) /$ Year $\left(\times 10^{-7}\right)$ \\
\hline AF & $1.8 \pm 0.3$ & $2.6 \pm 0.4$ \\
BP & $2.6 \pm 0.5$ & $3.8 \pm 0.8$ \\
RP & $3.8 \pm 0.3$ & $5.6 \pm 0.4$ \\
RVS & $3.1 \pm 0.5$ & $4.5 \pm 0.7$ \\
\hline \hline
\end{tabular}

destructive manner. This could be due to radiation interfering with the optics and photometers in some way, either causing secondary emissions or slowing down higher energy particles that may not have caused damage otherwise. In previous on-ground studies, it has been reported that aluminium shields in front of CCDs have caused higher energy particles to lose some of their kinetic energy so a similar phenomenon could be occurring here. ${ }^{19}$ Given only some BP devices measure this relatively higher increase though, this effect appears to be minimal, at least for the serial CTI.

From Tables 2 and 3, it can be seen that the rate of increase of CTI per year is around the order of magnitude of $10^{-7}$. This is at least two orders of magnitude smaller than the CTI values at the beginning of the mission, as noted in Table 1. This illustrates that the degradation of the CTI from the current status is very low.

\section{Parallel Charge Transfer Inefficiency (Along-Scan Direction)}

\subsection{4 and 2019 Data}

In a similar procedure to the serial CTI, the parallel CTI distribution of the CCDs across the focal plane in April 2014 was also reproduced, as given in Fig. 9(a) with Fig. 9(b) reproducing the year of manufacture plot from Fig. 2 for comparison. The results in Fig. 9(a) are similar to previous 

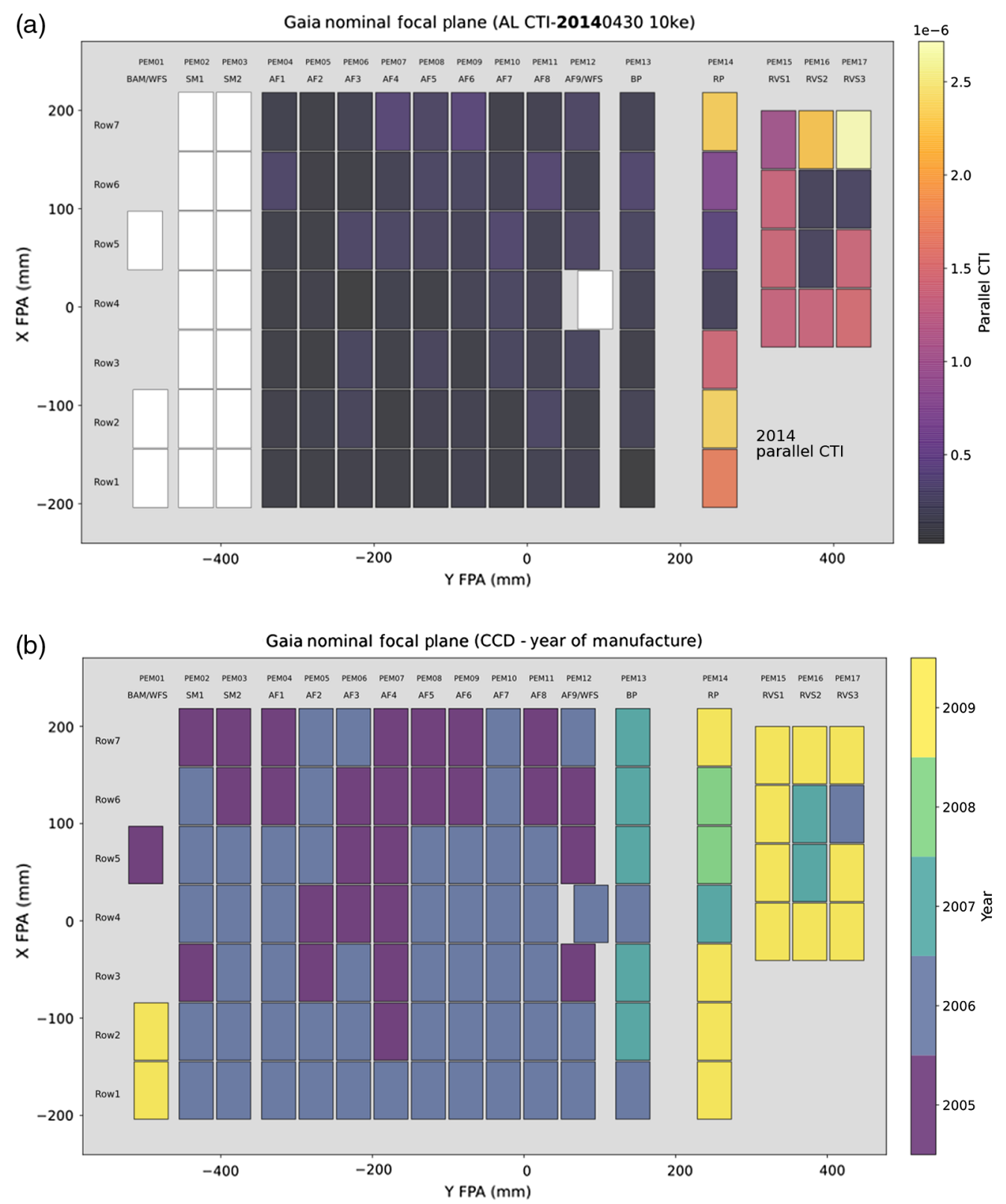

Fig. 9 (a) The distribution of the parallel CTI values across the focal plane as measured in April 2014 for a signal of 10,000 electrons reproduced from previous work. ${ }^{5}$ (b) A reproduction of Fig. 2 to compare against the parallel CTI results; the 2009 manufactured devices are measured to have a higher initial CTI.

CTI results that were taken for the CCDs before launch, both validating the data analysis procedure and confirming that the parallel CTI after launch is almost identical to the CTI before launch and extremely low. ${ }^{15}$

Comparing the parallel CTI results to the year of manufacture in both plots of Fig. 9 reveals that all the devices manufactured in 2009 measure a higher initial CTI as compared to the devices manufactured in earlier years. However, it is currently unknown why this may be. It could be due to a change in the manufacturing process or due to the use of a different silicon feedstock. This does not appear to be dependent on device variant as the RP devices manufactured in earlier years have comparable CTI values with the other device variants.

Figure 10 shows the distribution of the parallel CTI across the focal plane from the data taken in December 2019. This is several months past the original mission end date which means the results can be compared with the pre-flight tests to quantify the disparity between the two. As 


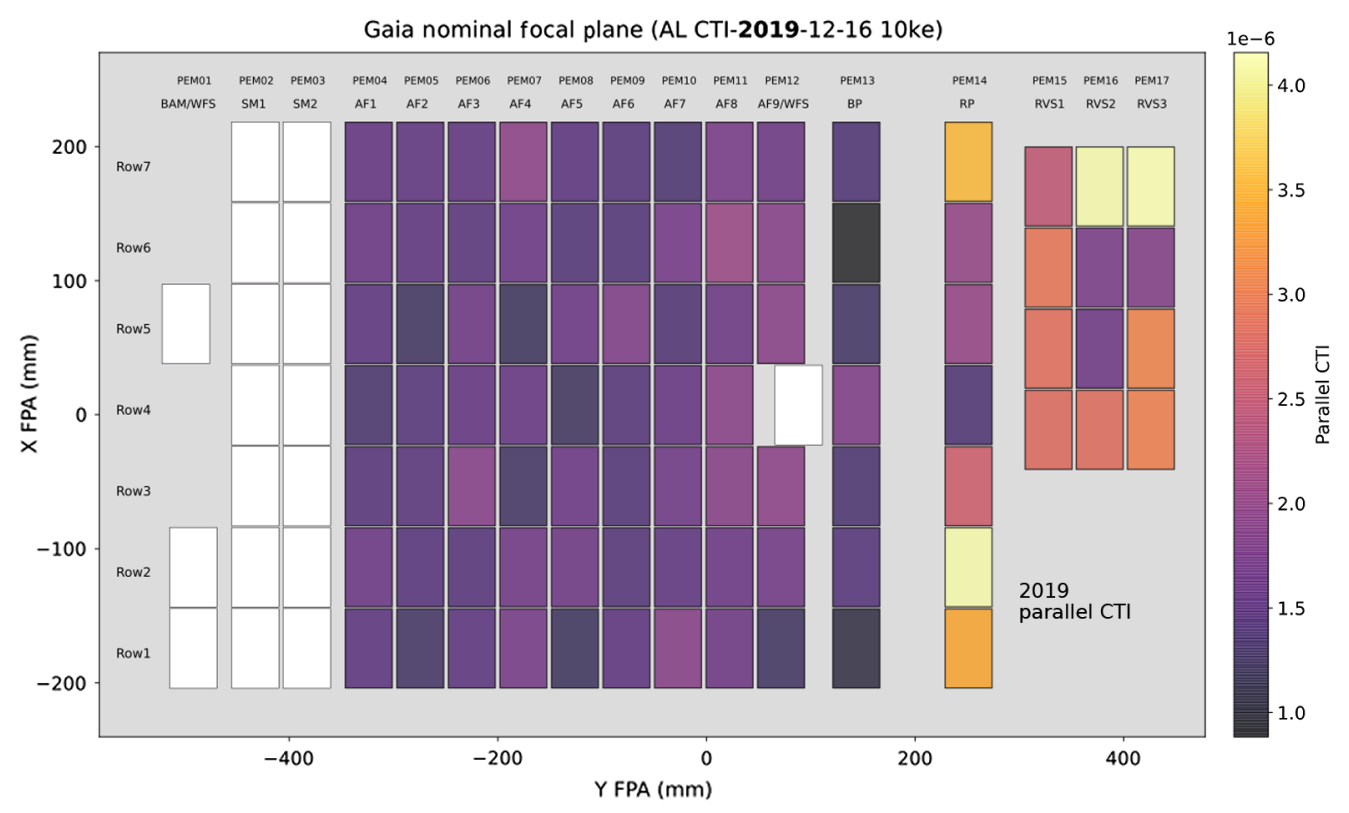

Fig. 10 The distribution of parallel CTI values across the focal plane as measured in December 2019 for a signal of 10,000 electrons; reproduced from previous work. ${ }^{5}$

Table 4 Mean parallel CTI (at 10,000 electrons) for each instrument as measured in April 2014 and December 2019.

\begin{tabular}{lcc}
\hline \hline CCD instruments & 2014 mean CTI $\left(\times 10^{-6}\right)$ & 2019 mean CTI $\left(\times 10^{-6}\right)$ \\
\hline AF & $0.17 \pm 0.09$ & $1.6 \pm 0.2$ \\
BP & $0.18 \pm 0.09$ & $1.3 \pm 0.3$ \\
RP (Pre-2009) & $0.4 \pm 0.2$ & $1.8 \pm 0.2$ \\
RP (2009) & $1.7 \pm 0.5$ & $3.6 \pm 0.6$ \\
\hline \hline
\end{tabular}

was the case for the serial CTI, the distribution pattern in 2019 is more reflective of the initial CTI state from 2014 than the predicted NIEL dose pattern. This suggests that the radiation damage has not been significant enough to drastically alter the CTI distribution across the focal plane. It also indicates that the long-term radiation damage contribution from the solar protons has been minimal as the NIEL pattern is not evident in Fig. 10. This lack of a clear NIEL pattern could also be due to the scale of the CTI values, which is limited by the red devices; previous studies have illustrated the pattern when studying just the AF devices. ${ }^{8}$

Table 4 gives the mean parallel CTI measurements for each instrument as measured in April 2014 and December 2019. To more reliably compare the devices against each other, the RP devices are split by their year of manufacture instead of by instrument. Comparing the 2014 CTI values in Table 4 to those in Table 1, it can be seen that the parallel CTI is up to two orders of magnitude lower than the initial serial CTI. In addition to this, the initial CTI measured by the 2009 devices is calculated to be higher by an order of magnitude as compared to the other devices.

The 2019 CTI values in Table 4 for each instrument and CCD variant are within the same order of magnitude with respect to each other. This again verifies that there is no apparent dependence between the initial parallel CTI and the rate of increase of CTI. However, the initial CTI is still affecting the 2019 CTI, as the 2009 devices measure the largest CTI values after six years. This highlights the lower level of radiation damage and demonstrates the need to keep track of the CTI at different stages of space missions and including on-ground testing and analysis to make sure every factor is accounted for. 


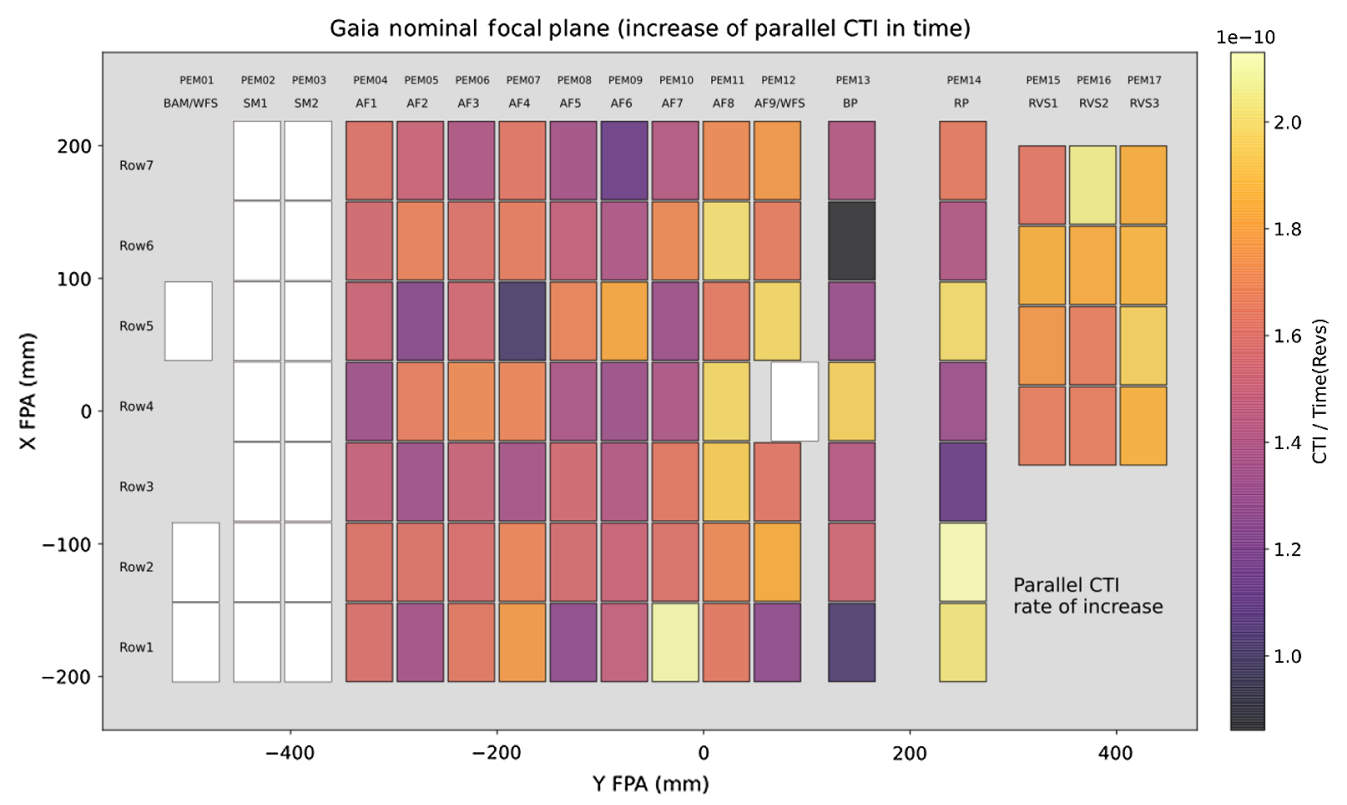

Fig. 11 The extrapolated rate of parallel CTI increase for each CCD as derived from the 6 years of charge calibration data, for a signal of 10,000 electrons.

\subsection{Rate of Increase}

As was conducted for the serial CTI, a linear relationship was extrapolated between the CTI for each CCD and time. Figure 11 shows the distribution of these rate of increase values across the focal plane. The NIEL-dose pattern across the AF devices from Fig. 3 is not present in Fig. 11; in previous results, the NIEL pattern has been visible across the focal plane for parallel CTI but only after the devices are investigated straight after the occurrence of a solar event. ${ }^{8}$ Parallel CTI degradation is most likely predominantly caused by other sources, such as higher energy GCRs; previous analysis of in-flight data have calculated that GCRs are responsible for around $75 \%$ of the parallel CTI degradation. ${ }^{9}$ The RP devices seem to measure slightly higher rates of increase compared to the other devices, possibly due to the reasons detailed previously.

Table 5 outlines the mean increase of CTI per instrument, also split by the year of manufacture for the RP devices to analyse effects of initial CTI. From Table 5, it can be seen that the CCDs with the higher initial CTI measure relatively the same rate of increase as the other devices. This demonstrates that the initial CTI does not affect the increase of CTI from radiationinduced traps; an expected result as the defects responsible for parallel CTI from manufacturing and radiation damage are known to be unique. The rate of parallel CTI increase for all the devices

Table 5 Mean rate of increase of parallel CTI increase for each instrument for a signal of 10,000 electrons. The RP variants are additionally split by their year of manufacture.

\begin{tabular}{lcc}
\hline \hline CCD instruments & CTI increase/revs $\left(\times 10^{-10}\right)$ & CTI increase/year $\left(\times 10^{-7}\right)$ \\
\hline AF & $1.6 \pm 0.2$ & $2.3 \pm 0.3$ \\
BP & $1.4 \pm 0.3$ & $2.0 \pm 0.5$ \\
RP & $1.7 \pm 0.4$ & $2.4 \pm 0.5$ \\
RVS & $1.8 \pm 0.1$ & $2.6 \pm 0.2$ \\
RP (pre-2009) & $1.7 \pm 0.2$ & $2.5 \pm 0.4$ \\
RP (2009) & $1.8 \pm 0.3$ & $2.6 \pm 0.4$ \\
\hline \hline
\end{tabular}




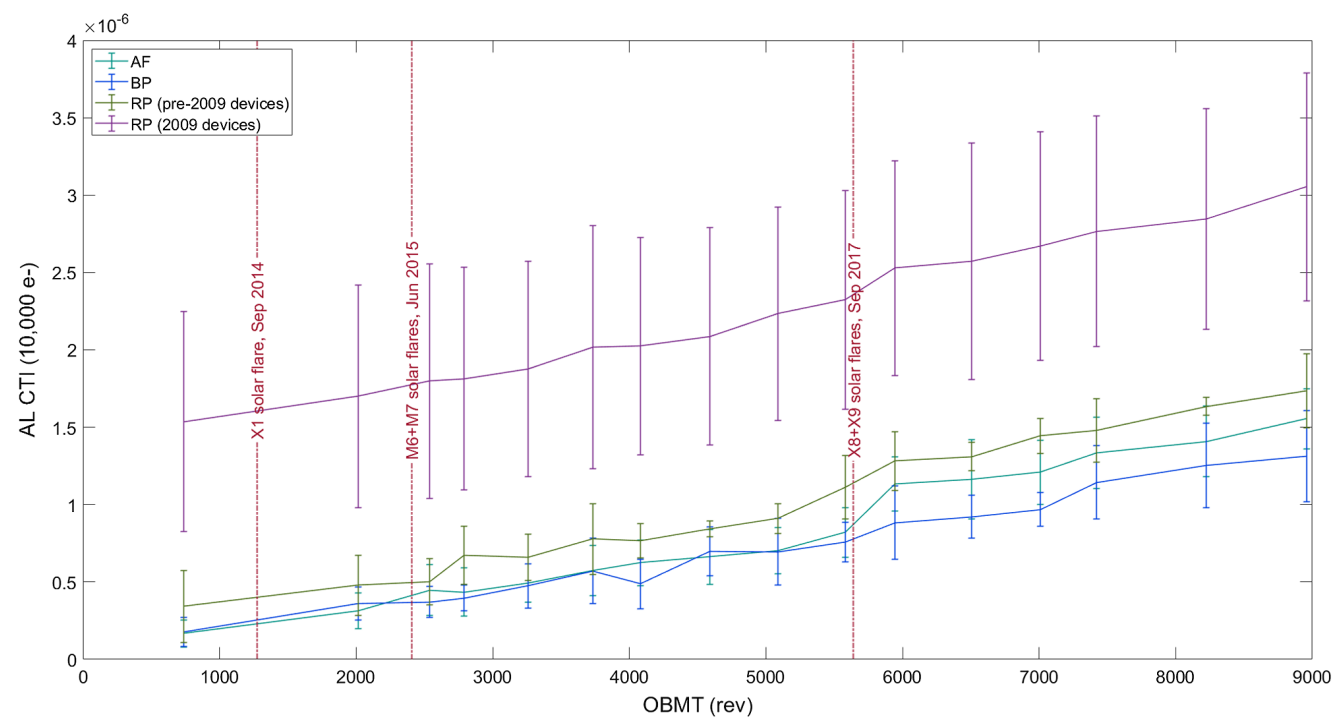

Fig. 12 Evolution of the mean parallel CTI (at 10,000 electrons) for each instrument, calculated from the CTI measured from all the devices at each different time. Instead of instrument, the thicker red devices are split by their year of manufacture. There is a steady, linear increase in time with fluctuations likely due to noise. Three main sets of solar flare events are all marked out. The $x$ axis spans a little under six years. The data from 04 November 2014 were omitted as it was defective for a number of devices.

is the same order of magnitude as it was for the serial CTI increase. This likely indicates that serial and parallel CTI are caused by different trap species that form independently of each other.

Figure 12 shows the mean CTI increase for the AF and BP instruments as well as the RP devices, split by their year of manufacture. Compared to results from previous studies, ${ }^{8,9}$ the impact of the solar flare events on the parallel CTI is less substantial. This could be due to the lower injection period and different signal values used in this data as well as a comparatively higher level of noise present in this dataset. The 2017 solar flare events cause a much more obvious impact on the CTI, with big step increases in the CTI for each instrument. The 2009 RP devices measure larger uncertainties in the CTI, likely due to the larger variance in the pre-flight trap defect concentrations and corresponding initial CTI values across fewer devices (as shown in Fig. 9), which seem to be more dominant over the radiation induced CTI. Similar to the serial CTI, a loosely linear increase of CTI in time is observed.

\subsection{NIEL-Scaled Rate of Increase}

To compare the CCD variants more reliably, the rate of increase of CTI for each CCD was scaled by the corresponding NIEL dose values from Fig. 3. This produces the distribution pattern as shown in Fig. 13; the values for each CCD were used to calculate the mean CTI increase values for each instrument as given in Table 6. Given that there was found to be no apparent dependence on the initial CTI in Table 5, the RP devices are not split by manufacture year in Table 6. From both Fig. 13 and Table 6, it can be seen that the BP and RP devices both measure higher CTI increases as compared to the AF devices, roughly by a factor of 2. Interestingly, both these sets of devices were predicted to have a lower $10-\mathrm{MeV}$ equivalent dose, as was seen in Fig. 3.

The $\mathrm{BP}$ devices are identical to the $\mathrm{AF}$ devices aside from a hafnium oxide coating to increase the quantum efficiency of the BP devices to bluer wavelengths and photometers setup in front of the devices. While no studies have looked into the effects of AR coatings on CTI, these are not believed to have a significant contribution. One current hypothesis is that the optics and photometers, alongside the higher energy GCR particles could be causing this increased damage in the BP devices, while also explaining the behavior of the RP devices. Hypothetically, particle energies could change when passing through the optical setups so there is more displacement damage per particle. 


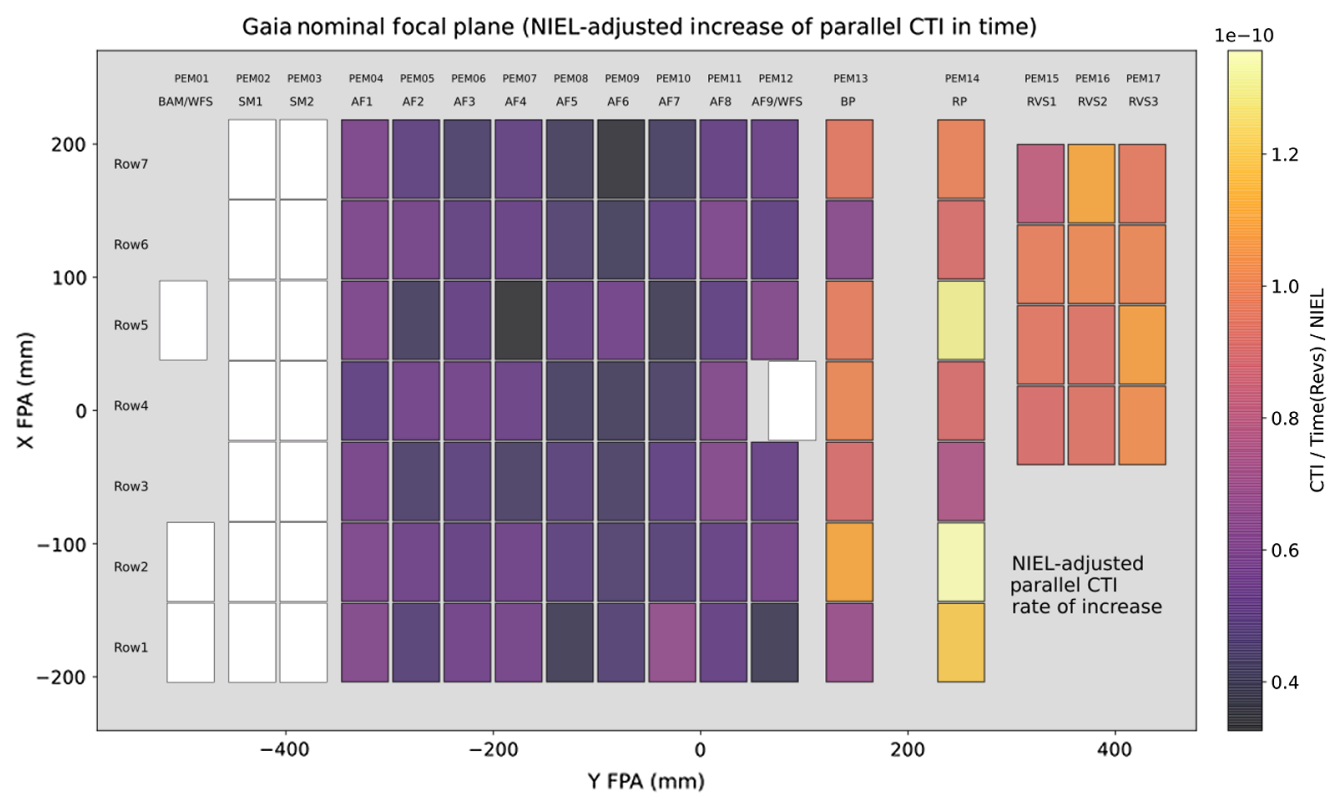

Fig. 13 The extrapolated rate of parallel CTI increase for each CCD as derived from the 6 years of charge calibration data, for a signal of 10,000 electrons, scaled by each CCD's corresponding NIEL-dose value from Fig. 3.

Table 6 Mean rate of increase of parallel CTI increase (at 10,000 electrons) for each instrument, scaled by the NIEL-dose values from Fig. 3.

\begin{tabular}{lcc}
\hline \hline CCD instruments & CTI increase (NIEL scaled)/Revs $\left(\times 10^{-10}\right)$ & CTI increase (NIEL scaled)/Year $\left(\times 10^{-7}\right)$ \\
\hline AF & $0.51 \pm 0.07$ & $0.7 \pm 0.1$ \\
BP & $0.9 \pm 0.2$ & $1.3 \pm 0.2$ \\
RP & $1.0 \pm 0.1$ & $1.5 \pm 0.2$ \\
RVS & $1.0 \pm 0.2$ & $1.5 \pm 0.2$ \\
\hline \hline
\end{tabular}

\subsection{Comparison with On-Ground Data}

In the previous analysis of in-flight data, comparisons were made with on-ground data taken from an irradiated flight-model CCD using the Gaia test bench at the ESA/ESTEC site in The Netherlands. ${ }^{9}$ By reproducing and extrapolating the results from the previous analysis (with permissions from the original author) and comparing them with the results of the in-flight calibration data from December 2019, it becomes possible to make another comparison between the on-ground and in-flight results. This is visualized in Fig. 14, with fractional charge loss measurements calculated as the total charge in the trailing pixels divided by the signal level (taken as the value of the final CI pixel).

For a signal of up to 10,000 electrons, the difference in fractional charge loss between the onground and in-flight results were found to be a factor of $\sim 20$ for the CCDs manufactured before 2009 and a factor of 11 for the CCDs manufactured in 2009. For larger signals around 40,000 electrons, the difference in fractional charge loss was found to be around 13 for the pre-2009 manufactured devices and a factor of around 9 for the 2009 devices. While it would appear as if the CCDs are functioning better than the factor of 7-8 difference measured in 2016, ${ }^{9}$ a number of elements are making this difference higher than it actually is. These reasons include the lower CI period as mentioned in Sec. 4 (as compared to the previous in-flight and on-ground periods) as well as the higher irradiation on the on-ground devices. After accounting for all the determinants, 
Ahmed et al.: Understanding the evolution of radiation damage...

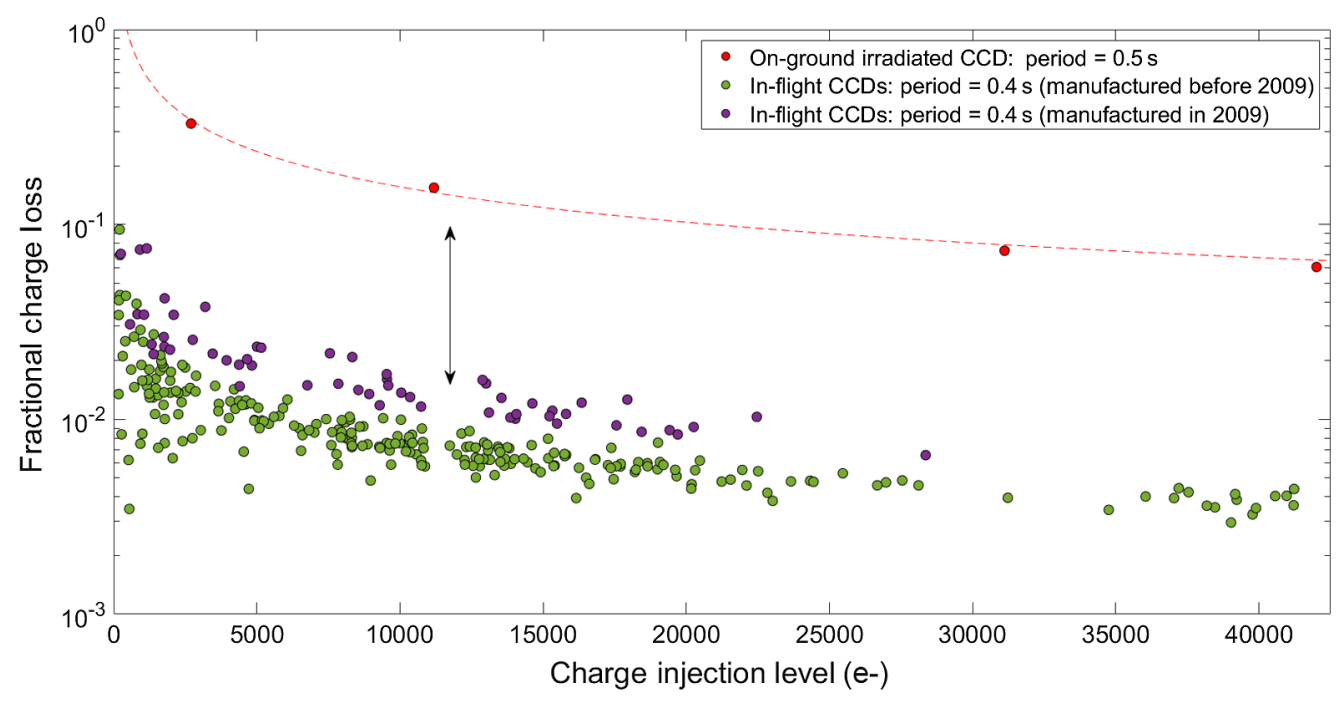

Fig. 14 A comparison of the fractional charge loss derived from the in-flight data taken at the end of 2019 with the charge loss values from an on-ground irradiated CCD, reproduced (with permissions) from the work done by Crowley et al. ${ }^{9}$ The lower signal-to-noise ratios in the lower Cls increases the variance in the values; error-bars have been omitted for visual clarity.

it is likely that the accumulated radiation damage has not deviated significantly from the predicted trend, extrapolated using the previous data in 2016.

\section{Solar Activity}

During the early pre-flight testing stage of Gaia, solar cycle predictions were made using the industrial standard, with $90 \%$ confidence levels, based on the current predictions of the behavior of Solar Cycle 24. ${ }^{14}$ However, as time went on, more predictions were made about a weaker solar cycle for the Sun instead. Figure 15 details the highest and lowest predicted activity levels for Solar Cycle 24, as predicted in 2009 as well as the actual activity, produced using data from the NOAA Space Environment Services Center. ${ }^{24,25}$ From the sunspot number, it can be seen that the

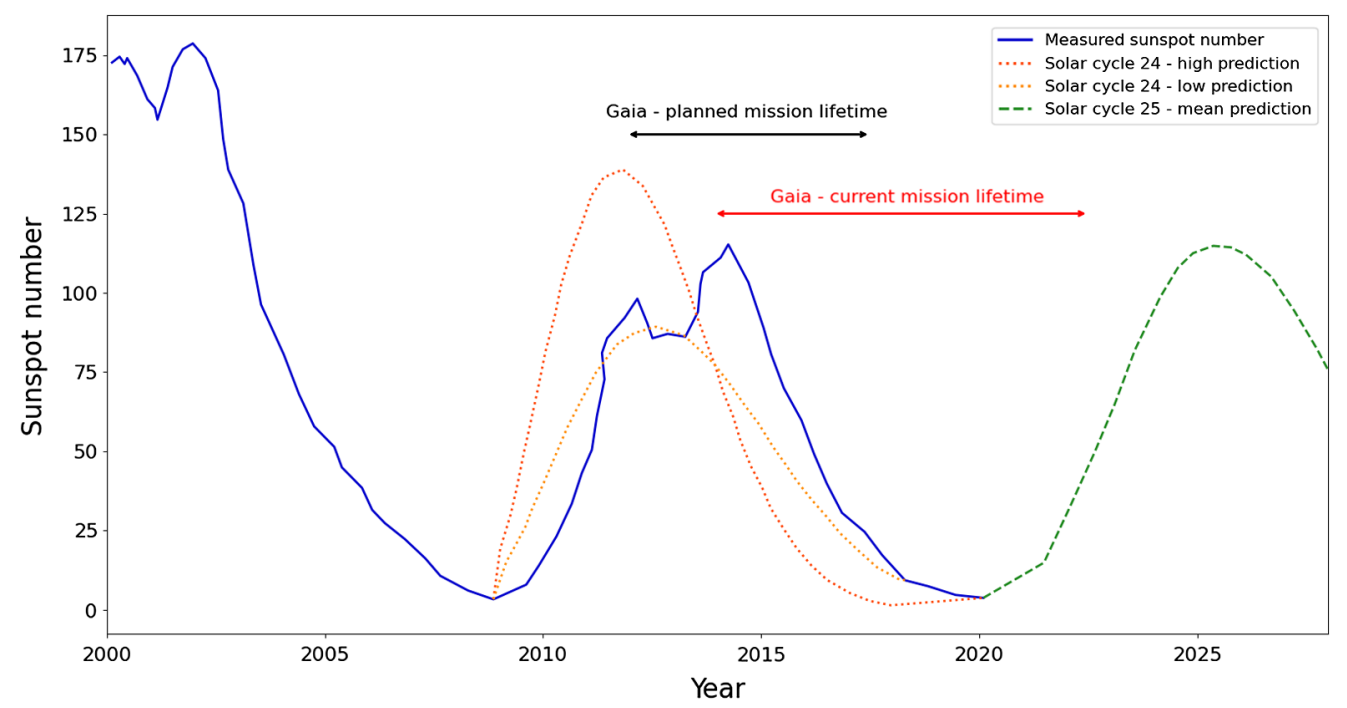

Fig. 15 The monthly mean sunspot number counts for the activity of the Sun from 2000 to 2020 compared with the high and low predictions of Solar Cycle 24 as well as the mean prediction for Solar Cycle 25 (proton flux source data: NOAA Space Environment Services Center). 
solar activity has been closer to the lower prediction level than to the highest. In addition to this, Gaia was planned to have been launched earlier, with the original mission timeline highlighted in Fig. 15, as prepared in 2010. Gaia was launched slightly later than its original proposed launch date and has a preliminary mission extension granted to 2022. Given the amount of fuel on board, Gaia could continue operating into 2024, over ten years since its launch date and double the original mission time, all due to the current exemplary levels of performance. ${ }^{26}$

Figure 15 shows an idea of how much the pre-flight predictions influenced the testing. Due to the earlier launch date and the $90 \%$ confidence levels for testing, pre-flight testing occurred with high irradiations of protons on comparative energy levels to solar protons. It is known that there is an anti-correlation between GCRs and solar protons where a lower flux of solar protons leads to a higher flux of GCRs. ${ }^{9}$ Due to the lower solar activity and nominal operations during the back half of Solar Cycle 24, the bulk of the radiation damage on Gaia has likely been a result of higher energy cosmic rays, as has been extrapolated from the analysis of these datasets.

It would be useful to keep track of more current predictions for solar cycles to have a more refined idea of the predicted radiation effects. Solar Cycle 25, to date, has been predicted to have approximately the same amount of activity as Solar Cycle 24, as shown in Fig. $15 .{ }^{25}$ As the Gaia mission has been extended, the spacecraft will now start to receive the effects of increased solar radiation from the beginning of Solar Cycle 25. In the AF devices, the effects of time-evolving CTI biases on astrometry are clearly visible and these effects on the AF CCDs in Data Release 3 (DR3) will be detailed in the documentation accompanying the release.

The CTI of the RP devices evolves relatively less in time compared to the AF and BP devices due to the higher initial CTI state. From DR 2, a slight CTI bias has been detected in the measurements of the RVS devices of the fainter magnitude stars when comparing results between Gaia data and data from ground-based catalogs. ${ }^{27}$ This CTI bias will affect DR3 radial velocities and is constant in time and will require empirical corrections; the CTI biases will be removed via posteriori calibrations. If the CTI bias is found to vary with time, more forward modeling techniques, such as CDM, or potentially an adaptation of a new model such as the CCD Charge Transfer Model (C3TM), ${ }^{28}$ will be required. Due to this, it is critical to keep track of the ongoing radiation damage in Gaia as the new solar cycle and successively increasing damage will lead to new sets of results.

\section{Discussion and Future Work}

Many interesting results and insights have been revealed from these results with respect to the correlations between CCDs and radiation damage. It is noted that the baseline for the periodic charge calibrations, as of the beginning of 2021, has been reduced to a cadence of approximately 6 months as opposed to the 3 to 4 months from the beginning of the mission. Increases in CTI from the nominal data are routinely monitored, and if a large damaging event is detected, additional sets of these calibrations will be run as needed.

It has also been noted that the pattern of the rate of increase of parallel and serial CTI has little similarity to the predicted $10 \mathrm{MeV}$ equivalent NIEL dose pattern, which used a solar proton spectrum as an input. From other studies, it has been calculated that higher energy GCRs have been responsible for the bulk of the radiation damage. ${ }^{8}$ The energy of the incoming particles could have an effect on the production of various trap defects. Alternatively, the cryogenic space environment could be causing different effects in the formation of trap species as compared with room temperature irradiations from the on-ground tests; this effect could be more pronounced in the parallel direction than the serial. ${ }^{9}$ To test these hypotheses, irradiations of different energies, and at different temperatures, should be performed on the same type of CCD, after which trappumping should be conducted to understand the trap-landscape and how it is affected by the different factors.

The rate of CTI increase is measured to be the same order of magnitude in the parallel and serial directions, of the order of around $10^{-7}$ a year. This would suggest that the trap species responsible for parallel and serial CTI are unique and not competing against each other. It is currently unknown why different devices at the beginning of the mission were measured to have different amounts of CTI, be it the lower serial CTI for all the RP devices or the higher initial 
parallel CTI for the RP devices manufactured in 2009. It is recommended to perform trap pumping between different sets of unirradiated devices, either thick and thin devices or the same devices manufactured at different times, to understand the trap-landscape differences between the device variants. This would provide a starting point into the nature of the trap defect map of CCDs of different architectures. It would also be useful to perform trap pumping on these same devices after irradiation as well, as the RP devices have been measured to have larger rates of increase than the other devices. This should provide more quantitative details on how different device variants are altered by radiation.

It has also been measured that the BP and RP devices measure relatively larger rates of increase (more evident in the parallel direction), than the AF devices. This is in contrast to the predicted $10-\mathrm{MeV}$ equivalent dose across the devices. The current hypothesis for this is that the photometers and optics setup in front of the BP and RP devices could be interfering with the incoming radiation. One hypothesis is that higher-energy particles are losing energy to the optics and hitting the detectors at a lower energy and hence causing more damage. This would line up with the conclusion that higher energy GCRs have been causing more damage, but specifically designed simulations (such as with Geant4) or laboratory tests would need to be conducted to verify this.

\section{Conclusions}

This paper has presented an overview of the CTI measurements of the science data measuring CCDs on-board Gaia over a six-year period. It has been shown that the overall rate of radiation damage accumulation has been relatively low since launch and the detectors are accumulating less damage than was predicted. The trap defect landscape is still dominated by defects present before launch after six years in orbit. Devices predicted to have a lower NIEL dose were measured to have comparatively larger rates of CTI increase. The RP devices that were manufactured in 2009 have also been measured to have comparatively higher levels of parallel CTI compared to earlier manufactured devices. All the thicker RP devices measured lower serial CTI values compared to the thinner device variants. While no definitive conclusions can be made, it has been suggested most of the damage has been due to higher energy particles which have a larger than expected flux due to the low solar activity. It has also been revealed that devices across the focal plane have different levels of CTI degradation but the exact physical reasoning behind this is currently unknown. Ultimately, more tests need to be conducted to get a better understanding of the CCD behavior; however, it can be said for certain that all the devices are operating well, and CTI levels of the Gaia CCDs are still well below pre-flight expectations as it undergoes mission extensions.

\section{Acknowledgments}

This work was carried out at the Center for Electronic Imaging; it would not have been made possible without access to in-flight data made available from the European Space Agency through a Networking Partnering Initiative. With additional thanks to the Science and Technology Facilities Council for their funding of the studentship and additional thanks to Teledyne e2v for sponsoring the CASE studentship.

\section{References}

1. M. Perryman, "Overview of the Gaia mission," in Proceedings of the Symposium on the Three-Dimensional Universe with Gaia, C. Turon, K. S. O'Flaherty, and M. A. C. Perryman, Eds., Vol. 576, p. 15 (2005).

2. T. Prusti et al., "The Gaia mission," Astron. Astrophys. 595, A1 (2016).

3. A. Brown et al., "Gaia data release 2 summary of the contents and survey properties," Astron. Astrophys. 616(1), A1 (2018).

4. ESA/Gaia/DPAC, "Gaia data," 2022, https://www.cosmos.esa.int/web/gaia/data (accessed 25 Janaury 2022). 
5. S. Ahmed et al., "Gaia CCDs: charge transfer inefficiency measurements between five years of flight," Proc. SPIE 11454, 114540S (2020).

6. S. W. Brown, "Characterization and mitigation of radiation damage on the Gaia astrometric field," PhD Thesis, University of Cambridge (2009).

7. T. Prod'homme, "From electrons to stars: modelling and mitigation of radiation damage effects on astronomical CCDs," PhD Thesis, Leiden University (2011).

8. C. Crowley et al., "Gaia data release 1-On-orbit performance of the Gaia CCDs at L2," Astron. Astrophys. 595, A6 (2016).

9. C. Crowley et al., "Radiation effects on the Gaia CCDs after 30 months at L2," Proc. SPIE 9915, 99150K (2016).

10. J. R. Janesick, Scientific Charge-Coupled Devices, Vol. 83, SPIE Press, Bellingham, Washington, DC (2001).

11. R. Kohley, F. Raison, and J. Martin-Fleitas, "Gaia: operational aspects and tests of Gaia Flight Model CCDs," Proc. SPIE 7439, 74390F (2009).

12. G. Seabroke, A. Holland, and M. Cropper, "Modelling radiation damage to ESA's Gaia satellite CCDs," Proc. SPIE 7021, 70211P (2008).

13. G. Seabroke et al., "Digging supplementary buried channels: investigating the notch architecture within the CCD pixels on ESA's Gaia satellite," Mon. Not. R. Astron. Soc. 430(4), 3155-3170 (2013).

14. R. Kohley et al., "The radiation environment at L2 as seen by Gaia," Proc. SPIE 9154, 915406 (2014).

15. C. Crowley, "An assessment of CCD radiation damage at launch +25 months," Tech. Rep. GAIA-CH-TN-ESA-CMC-017-1, ESAC (2016).

16. A. Short et al., "An analytical model of radiation-induced charge transfer inefficiency for CCD detectors," Mon. Not. R. Astron. Soc. 430(4), 3078-3085 (2013).

17. M. Cropper et al., "Gaia data release 2-Gaia radial velocity spectrometer," Astron. Astrophys. 616, A5 (2018).

18. D. J. Hall et al., "Challenges in photon-starved space astronomy in a harsh radiation environment using CCDs," Proc. SPIE 9602, 96020U (2015).

19. G. R. Hopkinson et al., "Radiation effects on astrometric ccds at low operating temperatures," IEEE Trans. Nucl. Sci. 52(6), 2664-2671 (2005).

20. S. E. Holland et al., "Fully depleted, back-illuminated charge-coupled devices fabricated on high-resistivity silicon," IEEE Trans. Electron Devices 50(1), 225-238 (2003).

21. D. J. Hall et al., "Determination of in situ trap properties in CCDs using a 'single-trap pumping' technique," IEEE Trans. Nucl. Sci. 61(4), 1826-1833 (2014).

22. D. Wood et al., "Studying charge-trapping defects within the silicon lattice of a p-channel CCD using a 'single-trap pumping' technique," J. Instrum. 9(12), C12028 (2014).

23. N. J. Murray et al., "The relationship between pumped traps and signal loss in buried channel CCDs," Proc. SPIE 8860, 88600H (2013).

24. NOAA Space Weather Prediction Center, "Solar cycle 24 prediction updated May 2009," 2009, https://www.swpc.noaa.gov/content/solar-cycle-24-prediction-updated-may-2009 (accessed 21 October 2020).

25. NOAA Space Weather Prediction Center, "Solar cycle progression," 2020, https://www .swpc.noaa.gov/products/solar-cycle-progression (accessed 21 October 2020).

26. E. Pancino, "Gaia: the galaxy in six (and more) dimensions," Adv. Space Res. 65(1), 1-10 (2020).

27. D. Katz et al., "Gaia data release 2-properties and validation of the radial velocities," Astron. Astrophys. 622, A205 (2019).

28. J. Skottfelt et al., "C3TM: CEI CCD charge transfer model for radiation damage analysis and testing," Proc. SPIE 10709, 1070918 (2018).

Saad Ahmed is a PhD student at the Open University. He received his BSc and MSc degrees in physics from Imperial College London in 2016 and 2017, respectively. He is currently analyzing the effects of radiation damage on the Gaia CCDs as part of the Center for Electronic Imaging, a collaboration between the Open University and Teledyne e2v. His current research interests 
include CCDs, radiation damage, instrumentation and design of space missions, astronomy, and astrometry.

David Hall studied physics at the University of Oxford before completing his $\mathrm{PhD}$ at the Open University, researching the impact of detection physics in X-ray CCD imagers and spectrometers. He is a research fellow in the Center for Electronic Imaging at the Open University. During the last 11 years, he has specialized in radiation damage in detectors and radiation induced defects in silicon, working on many ESA missions, including Gaia and Euclid.

Cian Crowley is an instrument operations scientist based at the European Space Astronomy Center. His $\mathrm{PhD}$ in observational astrophysics was carried out at Trinity College Dublin using, predominantly, ultraviolet spectral data. He has current interests in astronomical detectors, satellite payload operations and calibration, as well as the effects of radiation environments on payload components, in particular silicon detectors.

Jesper Skottfelt did his $\mathrm{PhD}$ in astronomy at the Niels Bohr Institute, Copenhagen University, Denmark. He was working on an electron multiplying CCD instrument and was responsible for the instrument control and data analysis software. He is currently a project officer in the Center for Electronic Imaging at the Open University, working on simulating charge transfer in radiation damaged CCDs to provide input to the radiation damage correction efforts for the Euclid mission.

Ben Dryer is a research fellow at the Center for Electronic Imaging at the Open University. He studied physics with industrial experience at the University of Bristol, followed by a $\mathrm{PhD}$ with the CEI. He is interested in the effects of radiation damage on space-bourne silicon imaging sensors with an emphasis on the impacts of cryogenic irradiations. He is also focused on the assessment of radiation damage effects for scientific operations through ground-based testing of image sensors.

George Seabroke studied Astronomy at University College London (UCL) before completing his $\mathrm{PhD}$ at the University of Cambridge, researching line-of-sight stellar radial velocities with the Radial Velocity Experiment (RAVE). He worked on radiation damage on Gaia at the Center for Electronic Imaging at the Open University. He is currently the scientific lead of the Mullard Space Science Laboratory (MSSL-UCL)'s Gaia team and Deputy Manager for Spectroscopic Processing in Gaia's Data Processing and Analysis Consortium (DPAC).

Jose Hernandez joined ESA in 1991 and he has been involved in the Science Operations of several ESA space telescopes (ISO, XMM-Newton, Integral) and some Planetary Missions. Since 2005, he has been part of the Gaia Science Operations team at ESAC, working in different aspects of the Gaia data processing. Currently, he leads the team in charge of producing the Core Astrometric Solution. His interests are in the area of scientific data processing of astronomical datasets and instrument calibration.

Andrew Holland is a professor in electro optics and head of the Center for Electronic Imaging, Open University. He is an expert in detector physics and has worked on the development of a number of successful space instruments. Working on a range of detector developments over the past two decades, he has a wealth of knowledge and experience advising on instrument related issues, in particular radiation damage effects. 